\title{
FORCED VIBRATION ANALYSIS OF EULER-BERNOULLI BEAM WITH STRESS STIFFENING OR WEAKENING BY MEANS OF DISTRIBUTIONS WITHOUT USING MODAL ANALYSIS
}

\begin{abstract}
J. Sobotka*
Abstract: The paper is the first continuation of previous author's work in which the author described the new approach to analyzing pre-stressed Euler-Bernoulli beam with discontinuities caused by concentrated loading, concentrated supports, concentrated inertia forces, or internal hinges situated between ends of the beam. The main advantage of this analytical approach is that it enables forced steady-state responses of the beam to be expressed exactly in closed forms. This way of dealing with discontinuities is applied here to obtain new closed-form solutions for another specific case of boundary conditions and discontinuous loading of the beam.
\end{abstract}

Keywords: vibration, beam, discontinuities, distributions, Dirac, Heaviside

\section{Introduction}

The new analytical method of computing forced steady-state response of pre-stressed Euler-Bernoulli beam with arbitrary combination of discontinuities of assumed types was described in (Sobotka, 2017). The discontinuities are supposed to be caused by concentrated supports, concentrated inertia masses, concentrated mass moments of inertia, concentrated transverse forces, concentrated moments, situated between ends of the beam, or by hinges connecting beam segments. This method has been based on using distributions (Schwartz, 1972; Štěpánek, 2001; Kanwal, 2004).

The new mathematical model for forced transverse vibration of the beam was derived by applying distributional derivative for discontinuous shear force, discontinuous bending moment, and discontinuous cross-section rotation. This mathematical model is solved as single differential task without dividing the beam into segments where all remaining continuity conditions among adjoining segments are fulfilled automatically. Applying this approach, we do not have to compute natural frequencies, mode shapes nor modal participation coefficients when analyzing forced responses of beams. Forced steady-state responses of the pre-stressed beam with various discontinuities can be expressed exactly in closed forms, which would be impossible if the modal analysis method was used.

The forced steady-state response of simply supported pre-stressed uniform beam with one concentrated transverse harmonic loading was published in (Sobotka, 2017). In this paper, new closed-form expressions for forced the steady-state response of pre-stressed beam are shown for another specific case of boundary conditions and discontinuous loading.

\section{The generalized mathematical model for forced steady-state transverse vibration of pre- stressed Euler-Bernoulli beam}

The beam is assumed to be fixed at its left end, and simply supported at the other end.

The beam is subjected to combination of a static axial force, $N$, and a concentrated transverse harmonic force $F \sin (\omega t)$ at $x=a$.

\footnotetext{
* Ing. Jiří Sobotka, Ph.D., ČEZ, a.s., 67550 Dukovany; CZ, e-mail: jiri.sobotka@cez.cz
} 
System of ordinary differential equations for unknown general amplitudes of shear force, $Q_{a}(x)$, bending moment, $M_{a}(x)$, cross-section rotation, $\phi_{a}(x)$, and deflection, $w_{a}(x)$, allowing for stress stiffening (in case of tensile axial load, $N>0$ ) or stress weakening (in case of compressive axial load, $N<0$ ) of the beam is as follows

$$
\begin{aligned}
\frac{d}{d x} Q_{a}(x)=-m w_{a}(x) \omega^{2}-N\left(\frac{d}{d x} \phi_{a}(x)\right)+F \delta(x-a), & , \\
\frac{d}{d x} M_{a}(x) & =Q_{a}(x), \\
\frac{d}{d x} \phi_{a}(x) & =-\frac{M_{a}(x)}{E J}, \\
\frac{d}{d x} w_{a}(x) & =\phi_{a}(x),
\end{aligned}
$$

where $E$ is modulus of elasticity (Young's modulus), $J$ is area moment of inertia, $m=\rho$ A, $\rho$ is density, A is cross-sectional area, $\omega$ is circular frequency of vibration, $0<a<l, l$ is length of the beam.

The right-hand side of Eq. (1) is the distributional derivative of shear force's amplitude with respect to $\mathrm{x}$, where $\delta(x-a)$ denotes Dirac's singular distribution moved to a point where the concentrated transverse load is acting. Dirac's singular distribution is multiplied by known magnitude of jump discontinuity.

Boundary conditions for fixed end of the beam are given by

$$
w_{a}(0)=0, \quad \phi_{a}(0)=0,
$$

Boundary conditions for simply supported end of the beam are given by

$$
w_{a}(l)=0, M_{a}(l)=0 \text {. }
$$

\subsection{The general solution to the system of Eqs. (1) to (4)}

Laplace transform method has been used to compute the general response of the beam in respect of bending moment (7) and deflection (8), where integration constants are in the form of initial parameters.

$$
\begin{aligned}
& M_{a}(x)=\frac{\left(\lambda_{1} \lambda_{2}{ }^{2} \sin \left(\lambda_{2} x\right)+\lambda_{1}{ }^{2} \lambda_{2} \sinh \left(\lambda_{1} x\right)\right) Q_{a}(0)}{\left(\lambda_{1}{ }^{2}+\lambda_{2}{ }^{2}\right) \lambda_{1} \lambda_{2}} \\
& +\frac{\left(\lambda_{1} \lambda_{2}{ }^{3} \cos \left(\lambda_{2} x\right)+\lambda_{1}^{3} \lambda_{2} \cosh \left(\lambda_{1} x\right)\right) M_{a}(0)}{\left(\lambda_{1}{ }^{2}+\lambda_{2}{ }^{2}\right) \lambda_{1} \lambda_{2}} \\
& +\frac{\left(m \omega^{2} \sin \left(\lambda_{2} x\right) \lambda_{1}-m \omega^{2} \lambda_{2} \sinh \left(\lambda_{1} x\right)\right) \phi_{a}(0)}{\left(\lambda_{1}{ }^{2}+\lambda_{2}{ }^{2}\right) \lambda_{1} \lambda_{2}} \\
& +\frac{\left(m \omega^{2} \lambda_{2} \lambda_{1} \cos \left(\lambda_{2} x\right)-m \omega^{2} \lambda_{2} \lambda_{1} \cosh \left(\lambda_{1} x\right)\right) w_{a}(0)}{\left(\lambda_{1}{ }^{2}+\lambda_{2}{ }^{2}\right) \lambda_{1} \lambda_{2}} \\
& +\frac{\left(-\lambda_{1} \lambda_{2}{ }^{2} \sin \left(\lambda_{2}(-x+a)\right)-\lambda_{1}{ }^{2} \lambda_{2} \sinh \left(\lambda_{1}(-x+a)\right)\right) F \mathrm{H}(x-a)}{\left(\lambda_{1}{ }^{2}+\lambda_{2}{ }^{2}\right) \lambda_{1} \lambda_{2}}
\end{aligned}
$$




$$
\begin{aligned}
& w_{a}(x)=\frac{\left(-\lambda_{2}{ }^{3} \lambda_{1}{ }^{2} \sinh \left(\lambda_{1} x\right)+\lambda_{2}{ }^{2} \lambda_{1}{ }^{3} \sin \left(\lambda_{2} x\right)\right) Q_{a}(0)}{\left(\lambda_{1}{ }^{2}+\lambda_{2}{ }^{2}\right) m \omega^{2} \lambda_{1} \lambda_{2}} \\
& +\frac{\left(\lambda_{2}{ }^{3} \lambda_{1}{ }^{3} \cos \left(\lambda_{2} x\right)-\lambda_{2}{ }^{3} \lambda_{1}{ }^{3} \cosh \left(\lambda_{1} x\right)\right) M_{a}(0)}{\left(\lambda_{1}{ }^{2}+\lambda_{2}{ }^{2}\right) m \omega^{2} \lambda_{1} \lambda_{2}} \\
& +\frac{\left(\lambda_{2}{ }^{3} \sinh \left(\lambda_{1} x\right) m \omega^{2}+\lambda_{1}{ }^{3} \sin \left(\lambda_{2} x\right) m \omega^{2}\right) \phi_{a}(0)}{\left(\lambda_{1}{ }^{2}+\lambda_{2}{ }^{2}\right) m \omega^{2} \lambda_{1} \lambda_{2}} \\
& +\frac{\left(\lambda_{2}{ }^{3} \cosh \left(\lambda_{1} x\right) m \omega^{2} \lambda_{1}+\lambda_{1}{ }^{3} \cos \left(\lambda_{2} x\right) m \omega^{2} \lambda_{2}\right) w_{a}(0)}{\left(\lambda_{1}{ }^{2}+\lambda_{2}{ }^{2}\right) m^{2} \omega^{2} \lambda_{1} \lambda_{2}} \\
& +\frac{\left(\lambda_{2}{ }^{3} \lambda_{1}{ }^{2} \sinh \left(\lambda_{1}(-x+a)\right)-\lambda_{2}{ }^{2} \lambda_{1}{ }^{3} \sin \left(\lambda_{2}(-x+a)\right)\right) F \mathrm{H}(x-a)}{\left(\lambda_{1}{ }^{2}+\lambda_{2}{ }^{2}\right) m \omega^{2} \lambda_{1} \lambda_{2}}
\end{aligned}
$$

where $\mathrm{H}(x-a)$ stands for Heaviside's unit step function, and where

$$
\begin{gathered}
m=\rho A, \quad \omega=2 \pi f \quad, \\
\lambda_{1}=\frac{\sqrt{2} \sqrt{\frac{N+\sqrt{N^{2}+4 E J m \omega^{2}}}{E J}}}{2}, \quad \lambda_{2}=\frac{\sqrt{2} \sqrt{\frac{-N+\sqrt{N^{2}+4 E J \omega^{2}}}{E J}}}{2} .
\end{gathered}
$$

\subsection{Determination of integration constants}

Two of four initial parameters are known directly as a consequence of boundary conditions (5). Eq. (7), (8) and boundary conditions (6) have been used in order to compute the remaining initial parameters (11), (12).

$$
\begin{gathered}
Q_{a}(0)=\frac{\left(-\cosh \left(\lambda_{1} l\right) \sin \left(\lambda_{2}(-l+a)\right) \lambda_{1}+\sinh \left(\lambda_{1}(-l+a)\right) \lambda_{2} \cos \left(\lambda_{2} l\right)\right) F}{-\sin \left(\lambda_{2} l\right) \cosh \left(\lambda_{1} l\right) \lambda_{1}+\lambda_{2} \cos \left(\lambda_{2} l\right) \sinh \left(\lambda_{1} l\right)}, \\
M_{a}(0)=\frac{\left(\sin \left(\lambda_{2}(-l+a)\right) \sinh \left(\lambda_{1} l\right)-\sin \left(\lambda_{2} l\right) \sinh \left(\lambda_{1}(-l+a)\right)\right) F}{-\sin \left(\lambda_{2} l\right) \cosh \left(\lambda_{1} l\right) \lambda_{1}+\lambda_{2} \cos \left(\lambda_{2} l\right) \sinh \left(\lambda_{1} l\right)} .
\end{gathered}
$$

\subsection{The deflection amplitude at the point of concentrated transverse load}

Since the right-hand side of Eq. (8) contains $\mathrm{H}(x-a)$, amplitude of deflection at $x=a$ has to be computed using bidirectional limit as follows

$$
\begin{aligned}
\lim _{x \rightarrow a} w_{a}(x)=\left(\left(\frac{1}{2} \cos \left(\lambda_{2} l\right) \cosh \left(\lambda_{1} l\right)-\frac{1}{2} \cosh \left(\lambda_{1} l\right) \cos \left(2 \lambda_{2} a-\lambda_{2} l\right)\right) \lambda_{2} \lambda_{1}{ }^{3}+(\right. \\
\quad-\frac{1}{2} \sin \left(\lambda_{2} l\right) \sinh \left(2 \lambda_{1} a-\lambda_{1} l\right)+\sin \left(\lambda_{2} l\right) \sinh \left(\lambda_{1} l\right)-2 \sin \left(-\lambda_{2} l+\lambda_{2} a\right) \sinh \left(-\lambda_{1} l+\lambda_{1} a\right) \\
\left.\quad-\frac{1}{2} \sinh \left(\lambda_{1} l\right) \sin \left(2 \lambda_{2} a-\lambda_{2} l\right)\right) \lambda_{2}{ }^{2} \lambda_{1}{ }^{2} \\
\left.+\left(\frac{1}{2} \cos \left(\lambda_{2} l\right) \cosh \left(2 \lambda_{1} a-\lambda_{1} l\right)-\frac{1}{2} \cos \left(\lambda_{2} l\right) \cosh \left(\lambda_{1} l\right)\right) \lambda_{2}{ }^{3} \lambda_{1}\right) F /\left(m \omega^{2}(\right. \\
\lambda_{1}{ }^{3} \sin \left(\lambda_{2} l\right) \cosh \left(\lambda_{1} l\right)-\lambda_{1}{ }^{2} \lambda_{2} \cos \left(\lambda_{2} l\right) \sinh \left(\lambda_{1} l\right)+\lambda_{2}{ }^{2} \sin \left(\lambda_{2} l\right) \cosh \left(\lambda_{1} l\right) \lambda_{1} \\
\left.\left.-\lambda_{2}{ }^{3} \cos \left(\lambda_{2} l\right) \sinh \left(\lambda_{1} l\right)\right)\right)
\end{aligned}
$$




\section{Conclusions}

In this paper, the generalized mathematical model published in 2017 for forced response of pre-stressed Euler-Bernoulli beam with discontinuities was applied to the specific case of loading and support conditions in order to derive new analytical solutions ready for direct use in practice.

Since the beam is subjected to concentrated transverse harmonic load, the distributional derivative of shear force (1) has to contain the respective jump discontinuity.

Amplitudes of shear force, bending moment, cross-section rotation and deflection of the beam have to satisfy Eqs. (1) to (4) and given boundary conditions (5), (6) for fixed left end and simply supported right end of the beam.

Eqs. (1) to (4) allow boundary conditions (5), (6) to be expressed directly without using derivatives of a deflection function.

The new closed-form expression (7) for the amplitude of bending moment with values of initial parameters corresponding to (5), (11), (12) was derived as the first contribution of this paper to analysis of forced vibration of pre-stressed beams.

The new closed-form expression (8) for the amplitude of deflection function with values of initial parameters according to (5), (11), (12) was obtained as the second contribution of the paper.

The new closed-form expression of the limit (13) for the amplitude of forced steady-state response of prestressed uniform beam at the point where concentrated transverse harmonic force is acting was computed as the third contribution of the paper.

All the new closed-form general and particular solutions would not be possible if modal analysis were used.

The right-hand side of Eq. (7), (8), (13) is exact in the sense of Euler-Bernoulli theory.

The effect of stress stiffening of the beam is covered by choosing a positive numeric value for axial tensile force, $N$, in Eqs. (7), (8), (13).

The effect of stress weakening is covered by choosing a negative numeric value for axial compressive force, $N$, not allowing buckling of the beam.

\section{References}

Kanwal, R. P. (2004) Generalized Functions. Birkhäuser, Boston.

Schwartz, L. and Brabec, J. (1972) Mathematical methods in physics. SNTL, Prague (in Czech).

Sobotka, J. (2017) Forced vibration analysis of pre-stressed Euler-Bernoulli beam with discontinuities by means of distributions without doing modal analysis, in: Engineering Mechanics 2017, Brno University of Technology, Brno, pp. 890-893.

Štěpánek, J. (2001) Distributions and differential equations. Karolinum, Prague (in Czech). 